\title{
Bound states in the continuum driven by $A C$ fields
}

\author{
C. González-Santander ${ }^{1}$, P. A. Orellana ${ }^{2}$ and F. Domínguez-Adame ${ }^{1}$ \\ 1 GISC, Departamento de Física de Materiales, Universidad Complutense, E-28040 Madrid, Spain \\ 2 Departamento de Física, Universidad Técnica Federico Santa María, Casilla 110 V, Valparaíso, Chile
}

PACS 73.22.-f - Electronic structure of nanoscale materials and related systems

PACS 73.63.-b- Electronic transport in nanoscale materials and structures

PACS 03.65.Nk - Scattering theory

\begin{abstract}
We report on the formation of bound states in the continuum driven by AC fields. The considered system consists of a quantum ring connected to two leads. An AC side-gate voltage controls the interference of electrons passing through the system. We model the system by two sites in parallel connected to two semi-infinite lattices. The energy of these sites changes harmonically with time. We obtain the transmission probability and the local density of states at the ring sites and show that transmission probability displays a Fano profile when the energy of the incoming electron approaches the driving frequency. Correspondingly, the local density of states presents a narrow peak that approaches a $\delta$-function in the weak coupling limit. We attribute these features to the presence of bound states in the continuum.
\end{abstract}

EPL (Europhysics Letters) 102, 17012 (2013)

Introduction. - At the dawn of quantum mechanics, von Neumann and Wigner constructed a spatially oscillating attractive potential that supported a bound state above the potential barrier [1]. This truly localized (square integrable) solution of the time-independent Schrödinger equation is referred to as a bound state in the continuum (BIC). Much later, Stillinger and Herrick reexamined and extended these ideas [2]. They analyzed a double excited atom model, where BICs were formed and had infinite lifetime despite the interaction between electrons. They arrived at the conclusion that BICs may be a physically realizable phenomenon in real atomic and molecular systems. In this context, Friedrich and Wintgen discussed a system of coupled Coulombic channels and, in particular, a hydrogen atom in a uniform magnetic field [3]. These authors interpreted the formation of BICs as the result of the interference between resonances of different channels. The observation of a BIC induced by the interaction between two particles in close proximity to an impurity has been recently reported by Zhang et al. [4].

The advent of nanotechnology has made it possible to devise and fabricate quantum devices whose electronic properties are similar to those of atoms and molecules. When the size of the device is comparable to the de Broglie wavelength, one or more degrees of freedom are quantized and the electron wave function is spatially confined. The similarity to atomic systems paved the way to experimentally validate the existence of BICs in artificial nanostructures. Capasso et al. measured the absorption spectrum at low temperature of a GaInAs quantum well with Bragg reflector barriers produced by a AlInAs/GaInAs superlattice [5]. A well defined line at $360 \mathrm{meV}$ in the spectrum was attributed to electron excitations from the ground state of the quantum well to a localized level well above the AlInAs band edge. Nevertheless, this state cannot be regarded as a true BIC but a bound state above the barrier since it is a defect mode residing in the minigap of the superlattice, as pointed out by Plotnik et al. [6]. More recently, Albo et al. used intersubband photocurrent spectroscopy to demonstrate that a BIC exists above $(\mathrm{Ga}, \mathrm{In})(\mathrm{As}, \mathrm{N}) /(\mathrm{Al}$.Ga)As quantum wells [7]. These BICs arise from the hybridization of nitrogen-related defect states and the extended states of the conduction band. Furthermore, it is worth mentioning that the analogy between photonic systems in 
the paraxial regime and electronic systems has facilitated the study [8-10] and subsequent experimental observation of BICs [6].

Electronic transport in mesoscopic and nanoscopic systems can be also influenced by the presence of BICs. Nöckel investigated theoretically the ballistic transport across a quantum dot in a weak magnetic field [11]. Resonances in the transmission were found to grow narrower with decreasing the magnetic field, and eventually they become BICs as the magnetic field vanishes. Fabry-Pérot interference of quasibound states of two open quantum dots connected by a long wire cause the occurrence of BICs [12]. The resulting state is nonlocal, in the sense that the electron is trapped in both quantum dots at the same time. Interestingly, controlling the size of one of the quantum dots makes the electron to flow or get trapped inside the dots. BICs were also found in parallel double quantum dot systems [13] and they were found to be robust even if electron-electron interaction is taken into account [14]. Recently, González et al. have demonstrated that not only quantum dots based on semiconductor materials but also on graphene can support BICs [15]. Furthermore, Dutta and Roy have shown that BICs may arise in heterogeneous nanostructures by engineering the spatial dependence of the effective mass of carriers [16]. All these features stimulate the interest of BICs to develop new applications in nanoelectronics.

In this work we extend the notion of BIC to the domain of time-dependent potentials. Our aim is twofold. First, we explore the possibility of the occurrence of BICs when the electronic system is driven by a time-harmonic potential. Second, we introduce a physical realizable system which opens a novel possibility to reveal the existence of these exotic states in transport experiments. As a major result, we show that the transmission, and correspondingly the conductance at low temperature, shows signatures of the occurrence of BICs. When the system is driven by an AC field the BICs survive and their existence is revealed by dynamic Fano resonances in the transmission probability. Remarkably, it turns out that their energy can be tuned by changing the frequency of the field. Therefore, the conductance at low temperature presents a minimum when the BIC crosses the Fermi level by varying the driving frequency.

Quantum ring under an AC side-gate voltage. -

The system under consideration is a two dimensional gas of noninteracting electrons in a quantum ring, shown schematically in Fig. 1a). The ring is connected to two leads (source and drain). A side-gate voltage $V_{ \pm}(t)$ breaks the symmetry of the upper and lower arms of the ring and acts as an additional parameter for controlling the electric current, as recently suggested for graphene-based nanorings $[17,18]$. We assume that the side-gate voltage can be modulated harmonically in time with frequency $\omega$. We are not considering capacitance effects as those described in Ref. [19] within the context of quantum rings threaded by an oscillating Aharonov-Bohm flux.

a)

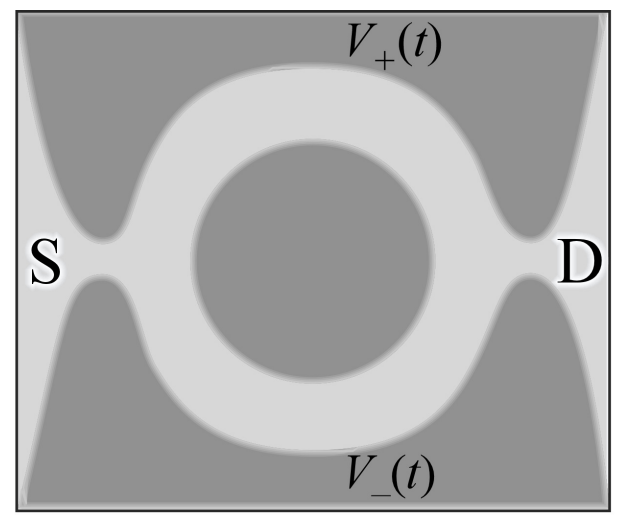

b)

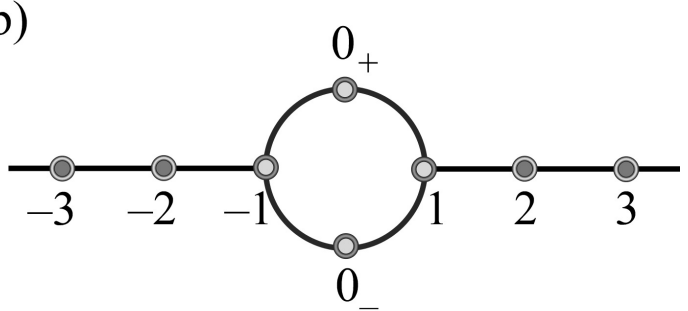

Fig. 1: a) Schematic diagram of the quantum ring with a side-gate voltage $V_{ \pm}(t)$ connected to source (S) and drain (D). b) Equivalent lattice model with two time-dependent site energies $\varepsilon_{ \pm}(t)$ at sites labeled $0_{ \pm}$and two other sites with index \pm 1 attached to semi-infinite chains.

In order to study electron transport across the quantum ring, we mapped it onto a much simpler yet nontrivial lattice model, depicted in Fig. 1b). We replace the actual quantum ring by four sites of a lattice within the tight-binding approximation. Two sites $\left(0_{ \pm}\right)$have timedependent energies $\varepsilon_{ \pm}(t)$ and the other two sites, labeled \pm 1 , are connected to semi-infinite chains. Time-dependent site energies are given by $\varepsilon_{ \pm}(t)= \pm 2 \Delta \cos \omega t$. To avoid the profusion of free parameters, we assume a uniform transfer integral and vanishing site energies except at sites $0_{ \pm}$, without loosing generality. The common value of the transfer integral will be set as the unit of energy and we take $\hbar=1$ throughout the paper.

Time-independent side-gate voltage. To gain insight into the possible occurrence of BICs in the system, we consider the time-independent case by setting $\omega=0$ for the moment. An incoming plane wave $\psi_{j}^{\text {in }}(t)=$ $\exp [i(k j-E t)]$, with energy $E=-2 \cos k$ within the bands of the leads, will be partially transmitted in the form $\psi_{j}^{\operatorname{tr}}(t)=t_{0} \exp [i(k j-E t)]$. The lattice period is set as the length unit. It is a matter of simple algebra to obtain the transmission amplitude in this case

$$
t_{0}=\frac{4 \sin k}{4 \sin k+i\left(E+4 \Delta^{2} / E\right)} .
$$

The transmission probability $T(E)=\left|t_{0}\right|^{2}$ presents a dip around the band center and vanishes at $E=0$. 
In the weak coupling limit, namely $\Delta \rightarrow 0$, the transmission probability shows a Fano profile [20] close to the band centre, $T(E)=E^{2} /\left(E^{2}+\Delta^{4}\right)$. The width of the dip scales as $\sim \Delta^{2}$. Poles of the transmission amplitude have a simple physical interpretation as the natural eigenstates of the scattering potential [21]. The poles occur at a complex energy whose real part gives the energy of the state and the imaginary part is related to its decay rate. There are four poles in the case under study. Two poles correspond to defect modes in the gap and, consequently, cannot be identified with BICs. However, the other two poles reside at the band centre with an imaginary part equal to $\pm i \Delta^{2}$ when $\Delta \ll 1$. In the weak coupling limit $\Delta \rightarrow 0$ the poles correspond to truly bound states. Therefore, the Fano resonance of the transmission amplitude (1) signals the occurrence of BICs at the band centre. A similar connection between features of the transmission probability and the energies of the electronic states of the closed ring was pointed out by Bütikker et al. when the ring is threaded by a static Aharonov-Bohm flux [22,23].

To get a better understanding of the nature of the BICs at the band center we also calculate the local density of states (LDOS) at sites $0_{ \pm}, \rho_{0}(E)$. Close to the band centre, the LDOS is proportional to $\left|\psi^{+}\right|^{2}+\left|\psi^{-}\right|^{2}$, where $\psi^{ \pm}$ is the wave function amplitude at those sites. The LDOS is given approximately as

$$
\rho_{0}(E) \sim \frac{E^{2}}{E^{2}+\Delta^{4}}+\frac{4 \Delta^{2}}{E^{2}+\Delta^{4}} .
$$

The first term is nothing but the transmission probability, vanishing at the band centre. However, the second term approaches $4 \pi \delta(E)$ in the limit $\Delta \rightarrow 0$, indicating the existence of a truly bound state with energy $E=0$ located at sites $0_{ \pm}$.

Time-dependent side-gate voltage. We now turn to our main goal, the occurrence of BICs when the sidegate voltage depends harmonically on time. The timedependent Schrödinger equation for the amplitudes $\psi_{j}(t)$ reads

$$
i \dot{\psi}_{j}=\varepsilon_{ \pm}(t) \delta_{j, 0_{ \pm}} \psi_{j}-\sum_{i(j)} \psi_{i(j)}
$$

where the index $i(j)$ runs over the nearest-neighbour sites of $j$ and the dot indicates the derivative with respect to time. Using the Floquet formalism, the solution can be expressed in the form

$$
\psi_{j}(t)=\sum_{n=-\infty}^{\infty} A_{n, j} e^{-i E_{n} t},
$$

where $E_{n}=E+n \omega$ and $n$ is the sideband channel index. Since we are interested in electron transmission across the ring, we take the following ansatz for the coefficients $A_{n, j}$ in the expansion (4a)

$$
A_{n, j}= \begin{cases}\delta_{n 0} e^{i k_{n} j}+r_{n} e^{-i k_{n} j}, & j \leq-1, \\ f_{n}^{ \pm}, & j=0_{ \pm} \\ t_{n} e^{i k_{n} j}, & j \geq 1 .\end{cases}
$$

Inserting this ansatz in (3) leads to the dispersion relation $E_{n}=-2 \cos k_{n}$ where $k_{n}$ is real if $E_{n}$ lies within the band, i.e. $|E+n \omega| \leq 2$. In addition, we obtain $t_{n}=f_{n}^{+}+f_{n}^{-}=$ $r_{n}+\delta_{n 0}$, ensuring current conservation. Finally, one also gets

$$
\alpha_{n} t_{n}-\frac{\Delta^{2}}{E_{n-1}} t_{n-2}-\frac{\Delta^{2}}{E_{n+1}} t_{n+2}=4 i \delta_{n 0} \sin k_{0},
$$

where for brevity we define

$$
\alpha_{n}=4 i \sin k_{n}-E_{n}-\Delta^{2}\left(\frac{1}{E_{n+1}}+\frac{1}{E_{n-1}}\right) .
$$

The continued fraction approach developed in Ref. [24] allows us to obtain numerically the contribution of all channels to the transmission. But if the coupling of the ring to the $\mathrm{AC}$ side-gate voltage is weak $(\Delta \ll 1)$, only the lowest order sidebands are significant. Then we keep five channels and assume that $t_{n}$ vanishes if $|n| \geq 3$. Equation (5) implies that $t_{ \pm 1}=0$ in this approximation and

$$
t_{ \pm 2}=\frac{\Delta^{2}}{\alpha_{ \pm 2} E_{ \pm 1}} t_{0}
$$

The transmission amplitude in the elastic channel is

$$
t_{0}=4 i \sin k_{0}\left[\alpha_{0}-\Delta^{4}\left(\frac{1}{\alpha_{2} E_{1}^{2}}+\frac{1}{\alpha_{-2} E_{-1}^{2}}\right)\right]^{-1}
$$

Once the transmission amplitudes have been calculated, we can obtain the transmission probability from the general expression

$$
T_{\omega}(E)=\sum_{n} \frac{\sin k_{n}}{\sin k_{0}}\left|t_{n}\right|^{2},
$$

where the sum runs over the propagating channels, namely those channels for which $E_{n}=E+n \omega$ lies within the band of the leads. Assuming that the sidebands $n= \pm 2$ are open, i.e. $k_{ \pm 2}$ are real, the transmission probability reads

$$
\begin{aligned}
T_{\omega}(E) & =\left|t_{0}\right|^{2}\left(1+\frac{\Delta^{4}}{\left|\alpha_{2}\right|^{2} E_{1}^{2}} \frac{\sin k_{2}}{\sin k_{0}}\right. \\
& \left.+\frac{\Delta^{4}}{\left|\alpha_{-2}\right|^{2} E_{-1}^{2}} \frac{\sin k_{-2}}{\sin k_{0}}\right) .
\end{aligned}
$$

Results. - The five channels approximation discussed above provides a closed analytical expression for the transmission probability (8). Admittedly the resulting expression is still involved and must be evaluated numerically for the chosen parameters. We will present some simpler expressions latter, valid in the weak coupling limit, but for the moment we are interested in the closed expression (8). We assume that the coupling $\Delta$ is smaller or on the order of the frequency $\omega$ hereafter. Figure 2 shows the results for $\omega=0.5$ and $\Delta=0.1$. The pronounced dip observed at the band centre in the static case $(\omega=0)$, 
commented above and shown in the figure, is absent if $\omega \neq 0$. Similar results can be obtained for other sets of $\Delta$ and $\omega$ parameters. In fact, transmission at $E=0$ is unity and the quantum ring becomes transparent at this energy. But the most salient feature of the transmission when the side-gate voltage oscillates is the occurrence of two symmetric and narrow dips, at energies close to $\pm \omega$. Remarkably, the transmission never vanishes in the range of energy plotted in Fig. 2 and at the dips only drops at about 0.5 . Actually, the transmission probability vanishes but only at the band edges, as occurs in the static case too. The inset shows an enlarged view of one of the dips for two different values of the coupling $\Delta$. It is quite apparent that the minimum transmission is slightly smaller than 0.5 and it is reached at an energy close but not exactly equal to $\omega$. In fact, transmission at $E=\omega$ is exactly equal to 0.5 for any value of $\Delta$.

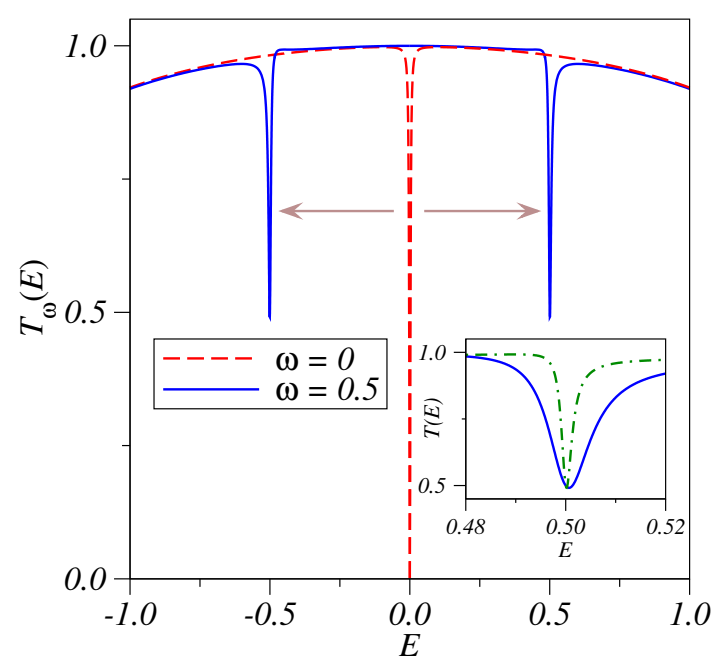

Fig. 2: Transmission probability as a function of energy at $\omega=0$ (red dashed line) and $\omega=0.5$ (blue solid line) for $\Delta=$ 0.1 . The inset shows an enlarged view of the transmission probability when $E \simeq \omega$ at $\Delta=0.01$ (green dashed line) and $\Delta=0.1$ (blue solid line) for $\omega=0.5$.

It is important to mention that we also solved numerically the general equation (5) to obtain the transmission probability from (7), increasing the number of the sideband channels. The plots were indistinguishable from those obtained within the five channels approximation, when $\Delta$ is smaller than $\omega$. Thus, we can confidently use this approximation in our analysis.

The transmission probability is an even function of energy. Therefore, for concreteness we now focus in the energy region close $+\omega$, when $\Delta$ is small. If $\omega$ is not large, we can take $\sin k_{ \pm 2} \simeq \sin k_{0} \simeq 1$ in (8). In addition, the term containing $\alpha_{2}$ in Eqs. (6b) and (8) is negligible under these assumptions. After lengthly but straightforward algebra, the transmission probability reduces to

$$
T_{\omega}(E) \simeq \frac{8(E-\omega)^{2}-\omega(E-\omega) \Delta^{2}+\Delta^{4}}{8(E-\omega)^{2}+2 \Delta^{4}} .
$$

Since $\omega$ and $E-\omega$ are not large, this expression can be further approximated by the following Fano profile

$$
T_{\omega}(E) \simeq \frac{1}{2}+\frac{1}{2} \frac{(x-\omega / 4)^{2}}{1+x^{2}}, \quad x=\frac{E-\omega}{\Delta_{\mathrm{eff}}^{2}} .
$$

To facilitate the comparison with the static case, we have introduced an effective coupling $\Delta_{\text {eff }}$ in such a way that $\Delta_{\text {eff }}^{2}=\Delta^{2} / 2$. Notice that the Fano factor $q=-\omega / 4$ becomes independent of the coupling $\Delta$. Figure 3 compares the exact results obtained from (8), for two different values of the coupling $\Delta$ and $\omega=0.5$, and the Fano profile (9b). In spite of the simplicity of the Fano profile and the assumptions we made, the agreement is remarkable in both cases.

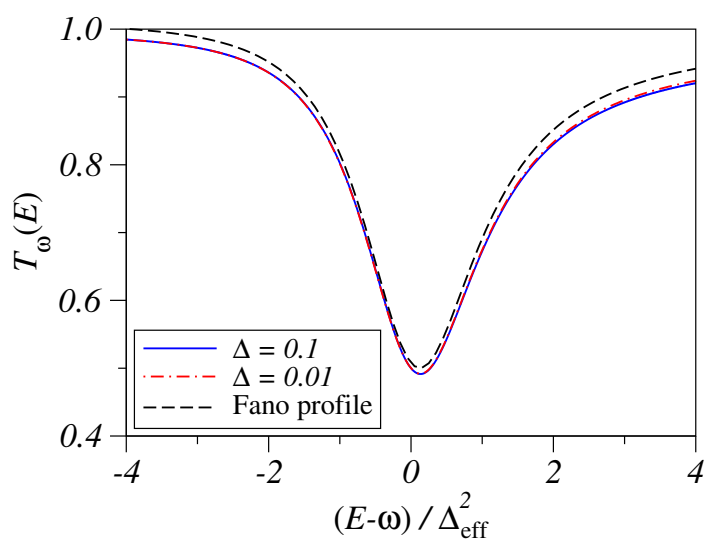

Fig. 3: Transmission probability as a function of the parameter $x$ defined in (9b), for two values of $\Delta$ and $\omega=0.5$. The Fano profile is a good approximation to both curves.

In contrast to the static case, transmission at the dips remains finite when the side-gate voltage is harmonically modulated in time (see Fig. 2). Therefore, it is not clear at this stage whether the dips are due to the occurrence of BICs in the system. To answer this question we consider again the LDOS at sites $0_{ \pm}$. After time-averaging over one time period, one gets

$$
\begin{aligned}
\overline{\rho_{0 \omega}}(E) & \sim \sum_{n}\left(\left|f_{n}^{+}\right|^{2}+\left|f_{n}^{-}\right|^{2}\right) \\
& =\frac{1}{2} \sum_{n}\left(\left|t_{n}\right|^{2}+\frac{\Delta^{2}}{E_{n}^{2}}\left|t_{n+1}+t_{n-1}\right|^{2}\right) .
\end{aligned}
$$

Using the five channels approximation, the LDOS close to right $\operatorname{dip}(E \sim \omega)$ becomes

$$
\overline{\rho_{0 \omega}}(E) \sim \frac{1}{2} T_{\omega}(E)+\frac{\Delta_{\text {eff }}^{2}}{(E-\omega)^{2}+\Delta_{\text {eff }}^{4}},
$$

and a similar expression is obtained for the left $\operatorname{dip}(E \sim$ $-\omega)$, replacing $\omega$ by $-\omega$ in (10). Therefore, in the weak coupling limit $\Delta \rightarrow 0$, the LDOS reduces to $\overline{\rho_{0 \omega}}(E) \sim$ $\delta(E+\omega)+\delta(E-\omega)$. In analogy with the static case, we claim that the two singular peaks in the LDOS at energies $\pm \omega$ are due to a new type of BICs arising by the interaction with the AC field. 
Conclusions. - In summary, we have introduced and studied a novel type of BICs in systems whose energy levels are modulated harmonically in time. To be specific, we have considered a quantum ring subjected to a side-gate voltage oscillating in time with frequency $\omega$ and studied its transport properties in a fully coherent regime. We come to the important conclusion that the BICs supported by the quantum ring in the static case survive under harmonic modulation of the side-gate voltage. The two BICs driven by the AC field have energies $\pm \omega$ and they reveal themselves in the transmission, and consequently in the lowtemperature conductance, as dynamic Fano resonances. The position of the BICs inside the spectral band can be continuously tuned by varying the driving frequency and eventually they could be expelled out of the continuum when $\omega$ is larger than 2 in units of the transfer integral, i.e. when they approaches the band edge. Similar control of the energy of static BICs have been recently demonstrated by adding weak nonlinearity to semi-infinite systems [25] or by varying the interaction between particles [4]. However, besides the different origin of the BICs, our proposal seems to be more advantageous for the experimental validation of these exotic states. In this regard, it is still an open question to what extend electron-electron interactions would mask the effect in a real experiment. Žtiko et al. have shown that the so-called dark states in parallel double quantum dot systems are robust against interactions within a Hubbard model, at least in the Kondo regime [14]. These states correspond to the BICs shown in the present work when $\omega=0$, which makes us confident to expect that BICs driven by $\mathrm{AC}$ fields are robust even if interactions are taken into account.

$$
* * *
$$

The authors thank A. V. Malyshev for a critical reading of the manuscript. Work at Madrid was supported by MICINN (project MAT2010-17180). C. G.-S. acknowledges financial support from Comunidad de Madrid and European Social Foundation. P. A. O. acknowledges financial support from FONDECYT under grant 1100560.

\section{REFERENCES}

[1] von Neumann J. and Wigner E., Phys. Z., 30 (1929) 465.

[2] Stillinger F. H. and Herrick D. R., Phys. Rev. A, 11 (1975) 446.

[3] Friedrich H. and Wintgen D., Phys. Rev. A, 31 (1985) 3964.

[4] Zhang J. M., Braak D. and Kollar M., Phys. Rev. Lett., 109 (2012) 116405.

[5] Capasso F., Sirtori C., Faist J., Sivco D. L., Chu S. and Chо A. Y., Nature, 358 (1992) 565.

[6] Plotnik Y., Peleg O., Dreisow F., Heinrich M., Nolte S., Szameit A. and Segev M., Phys. Rev. Lett., 107 (2011) 183901.
[7] Albo A., Fekete D. and Bahir G., Phys. Rev. B, 85 (2012) 115307.

[8] Longhi S., Eur. Phys. J. B, 57 (2007) 45.

[9] Moiseyev N., Phys. Rev. Lett., 102 (2009) 167404.

[10] Bulgakov E. N. and Sadreev A. F., Phys. Rev. B, 81 (2010) 115128.

[11] Nöckel J. U., Phys. Rev. B, 46 (1992) 15348.

[12] Ordonez G., Na K. and Kim S., Phys. Rev. A, 73 (2006) 022113.

[13] Rajput G., Ahluwalia P. K. and Sharma K. C., Europhys. Lett., 94 (2011) 17003.

[14] Žitko R., Mravlje J. and Haule K., Phys. Rev. Lett., 108 (2012) 066602.

[15] González J. W., Pacheco M., Rosales L. and OreLlANA P. A., Europhys. Lett., 91 (2010) 66001.

[16] Dutta D. and Roy P., Europhys. Lett., 89 (2010) 20007.

[17] Munárriz J., Domínguez-Adame F. and Malyshev A. V., Nanotechnology, 22 (2011) 365201.

[18] Munárriz J., Domínguez-Adame F., Orellana P. A. and Malyshev A. V., Nanotechnology, 23 (2012) 205202.

[19] Büttiker M., Physica Scripta, 1994 (1994) 104.

[20] Fano U., Phys. Rev., 124 (1961) 1866.

[21] Bagwell P. F. and Lake R. K., Phys. Rev. B, 46 (1992) 15329.

[22] Büttiker M., Imry Y. and Azbel M. Y., Phys. Rev. A, 30 (1984) 1982.

[23] Taniguchi T. and Büttiker M., Phys. Rev. B, 60 (1999) 13814.

[24] Martinez D. F. and Reichl L. E., Phys. Rev. B, 64 (2001) 245315.

[25] Molina M. I., Miroshnichenko A. E. and Kivshar Y. S., Phys. Rev. Lett., 108 (2012) 070401. 